\title{
Fate and impact of pesticides: new directions to explore
}

\author{
Céline Pelosi $^{1} \cdot$ Enrique Barriuso ${ }^{2} \cdot$ Carole Bedos $^{2} \cdot$ Pierre Benoit $^{2} \cdot$ Laure Mamy $^{2}$. \\ Christian Mougin $^{1}{ }^{1}$
}

Received: 27 December 2016 / Accepted: 3 January 2017 / Published online: 27 February 2017

(C) Springer-Verlag Berlin Heidelberg 2017

The consequences of the environmental contamination by pesticides on human and ecosystem health are of major concern. A strong research effort is needed to better understand the deleterious effects of pesticides, to reduce uncertainties concerning these potential effects, and to inform public policies and citizens. This effort has to focus on different scientific fields of research such as toxicology, ecotoxicology, epidemiology, and human and social sciences. The finality is to propose solutions to reduce the risks related to the dispersion of pesticides in the environment and to decrease their harmful impacts on biodiversity, ecosystem functions, and human health.

Improving the human and ecosystem exposure measurements remains a challenge for pesticide risk assessment strategies. Standardized and integrated protocols are needed for in situ monitoring of pesticides in various ecosystems. This can allow for more realistic ecotoxicological studies as well as epidemiological surveys on the impacts of pesticides on the environment and human health. Integrating new results at different levels of organization including human activities is critical to address the difficult question of long-term effects of pesticides at low concentrations and for compound mixtures. It is therefore necessary to rely on new tools and methods, e.g., sensors, biomarkers, bio-omics, analytical platforms,

Responsible editor: Philippe Garrigues

Christian Mougin

christian.mougin@inra.fr

1 UMR ECOSYS, INRA, AgroParisTech, Université Paris - Saclay, 78026 Versailles, France

2 UMR ECOSYS, INRA, AgroParisTech, Université Paris - Saclay, 78850 Thiverval-Grignon, France dedicated to ecotoxicological approaches, predictive toxicology, and epidemiological studies.

Designing cropping systems to reduce pesticide use and/or impacts at different spatial scales and levels of organization has become a clear research orientation in Europe. In France, the Ecophyto plan called for a decrease in pesticide use. Although this objective is far to be reached, this recent policy has launched many research programs in the last 10 years. Significant progresses have been made regarding the conception and assessment of low input cropping system performances. Alternative agricultural practices such as conservation tillage, selected crop varieties, biological regulations of bioagressors at the plot, farm, and landscape scales may significantly contribute to decrease the pesticide pressure.

Improving our knowledge of pesticide transfers outside from the treated plots remains necessary before promoting measures to reduce pesticide dispersion at the watershed scale. Local processes regulating the interactions between the soil, air, vegetation, and water have to be thoroughly understood. Field observations, experimental studies, and modeling approaches addressing the dispersion of pesticides should consider both the plot and catchment scales in a variety of landscapes - from rural to peri-urban areas - and under a variety of pedoclimatic conditions. Ecological infrastructures such as buffer zones can be used in these different landscapes to reduce the dispersion. However, a quantitative assessment of their efficiency and the integration of their functioning in the pesticide transfer models are required.

Modeling pesticide fate and transport as well as their biological effects on living organisms requires a detailed knowledge of physicochemical and biological processes at microscales. These processes occur in soil porosity colonized by soil microorganisms, within plant organs and tissues, at the interface between soil, plant, air, or water. Innovative approaches for the study of these processes coupled to the 
development of models at these microscales can bring new insights to our understanding at macroscopical scale.

Finally, modeling the processes governing pesticide fate and effects also requires considering not only pedoclimatic parameters but also various drivers such as the cropping practices or molecular properties of pesticides. Therefore, upscaling models from local processes to the plot scale where cropping practices have a direct impact on the soil-plantatmosphere-water compartments is required. Such an integrative approach can be achieved by combining different numerical models under modeling platforms. This allows to offer different coupling options and to cover a broader diversity of cropping systems and chemicals (in terms of behavior, mode of action, and ecotoxicological and toxicological effects).

As previously mentioned, pesticide use is at the origin of societal issues involving several factors. Studies focusing on pesticides and accounting for human and social sciences allow for a better understanding of the perception of pesticide risk by different groups, such as farmers and citizens. It can help to adapt regulatory decisions or to promote co-learning actions leading to the acceptation of protection measures to reduce pesticide exposure for different groups of citizens.

Since 1977, francophone researchers working on pesticides get together for the annual symposium of the "Groupe Français des Pesticides" association (GFP). GFP is an association aiming at gathering scientific community coming both from the private and public sectors, involved in the study of pesticides whatever the disciplines, leading to transversal views and then innovative initiatives. In 2015, the 45th symposium was held in the INRA centre in Versailles, from May 27 to 29. It was granted by INRA (EA and SPE divisions), the federation FIRE, the LabEx BASC, ONEMA, French Ministries (MAAF, MEEM), and the companies Thermo Scientific and Cluzeau Info Labo. Scientists from French (including overseas) and foreign universities and higher schools, but also from French Public Research Institutes (ANSES, BRGM, CNRS, CEA, Cirad, IFREMER, INRA, INSERM, IRD, and IRSTEA) and from the industry came for exchanging their views and respective expertise on scientific news. The event was organized by ECOSYS INRAAgroParisTech Joint Research Unit (functional ecology and ecotoxicology of agroecosystems). The main research object of ECOSYS is the functioning of agroecosystems considered in an integrated way. To understand this functioning, the ECOSYS unit works with concepts of functional ecology and ecotoxicology, taking into account the flux of matter and energy and the functions of isolated organisms and the interaction with their environment. One research topic concerns the assessment of the environmental impacts of pesticide use in agriculture and gathers different scientists working in the domain of pesticide emission and transport in the atmosphere, soil processes including sorption, biodegradation and transport, ecotoxicological impacts on soil biota (mesofauna, macrofauna, and soil microflora), and modeling.

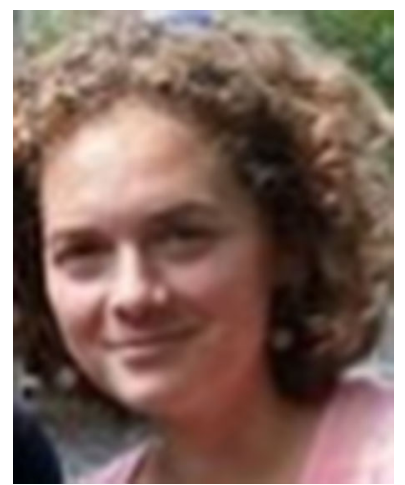

Céline Pelosi is a scientist at INRA in the "Functional Ecology and Ecotoxicology of Agro-Ecosystems" (ECOSYS) Joint Research Unit in Versailles, France. Her research aims to assess and model the consequences of pesticide use on soil organisms, from their application in the field to their functional consequences on the ecosystems. To improve risk assessment procedures, and influenced by her background in agroecology, she is currently working at developing a more integrated and relevant approach to soil ecotoxicology in agroecosystems. For that, her research combines field observations, laboratory experiments, and modeling to gather ecological, agronomic, and ecotoxicological tools and concepts.

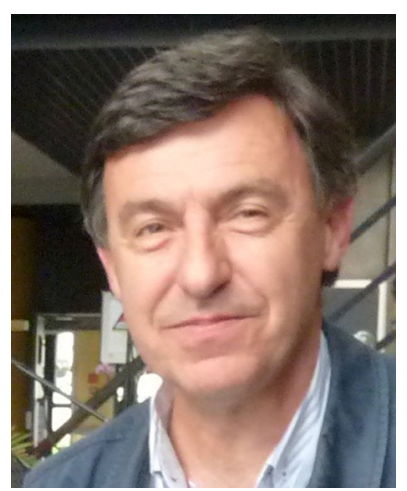

Enrique Barriuso is a senior scientist at INRA specializing on environment fate of pesticides. He works on physicochemical and chemical processes responsible for pesticide sorption/desorption and the formation of bound residues. He was a scientific coordinator of Pesticides program of the French Ministry of the Environment, and he is actually a member of scientific committee of the research axis of the Ecophyto plan. He is actually the head of the INRA-AgroParisTech Joint Research Unit ECOSYS, whose main goal is to describe and model the functioning of agricultural systems that are representative of arable crops in northern Europe in terms of their interactions with biotic and abiotic environmental factors (climate, soil, pollutants, and pathogens). The objective is to draw up assessments of the positive and negative impacts of agronomic practices and other technical decisions, as pesticides use, on the quality of different environmental compartments (http://www6.versailles-grignon.inra.fr/ecosys/). 


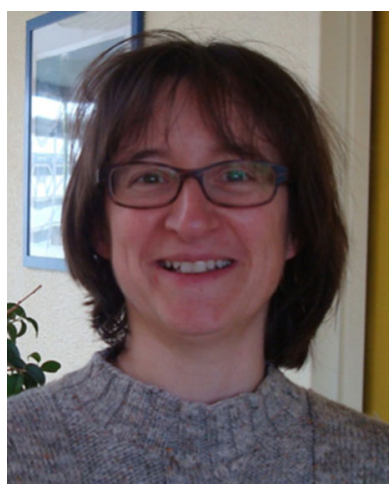

Carole Bedos is working as a researcher at the Joint Research Unit ECOSYS since 1999. Her main research interests are pesticide emission to the atmosphere through volatilization and recently gaseous deposition on nontarget ecosystems close to treated fields. Approaches are based on experimental studies with laboratory systems specially designed to analyze the determinism of the emission and/or deposition processes as well as micrometeorological methods at the field scale. Operational tools are provided by modeling developments. Current projects aim at understanding the level of contamination of the atmosphere by pesticide at a regional scale.

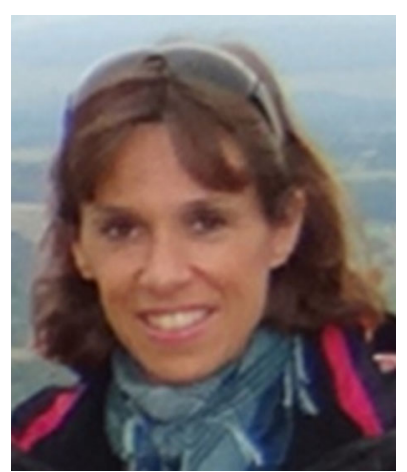

Laure Mamy is a scientist at INRA in the ECOSYS Joint Research Unit. Her research mainly focuses on the modeling of the fate of pesticides in the environment. One of the main objectives of the projects she currently coordinates is to develop new modeling approaches to assess the impacts of the use of pesticides in complex cropping systems to a priori identify the most efficient systems. Her research also aims at understanding the molecular properties that drive the fate of pesticides in the environment, and she has a strong interest in quantitative structure-activity relationships (QSAR). In parallel, she is an environmental fate expert for the French Agency for Food, Environmental and Occupational Health and Safety (ANSES), working on environmental risk assessment for pesticides registration.

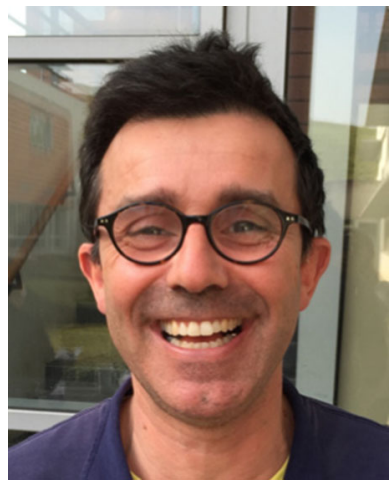

Pierre Benoit is currently the leader of the Soil Science Group (30 permanent staff) at the Joint Research Unit ECOSYS. His research interests concern soil science and the coupling of biological and physicochemical processes involved in the fate and transport of pesticides and other potential contaminants in soil and water. Applications concern the assessment of several management and cropping practices such as organic waste recycling in agricultural soils, soil tillage practices, cover crop introduction, installation of grassed buffer strips, and their impacts on the fate and transport of pesticides and other organic contaminants. $\mathrm{He}$ has in the past coordinated one international project on the effect of climate change on pesticide fate in Nordic regions in collaboration with Bioforsk (Norway). He also coordinated another project with Mexico dealing with the fate of insecticides in tropical soils and their impacts of earthworms. Recently, he has coordinated a national collaborative project which proposed an operational tool for the in silico classification of pesticides and other organic contaminants (Typology of Pollutants (TyPol)). This tool is based on statistical analyses combining several environmental endpoints and structural molecular descriptors and allows obtaining clusters of compounds, including pesticide metabolites, according to their potential behaviors in the environment.

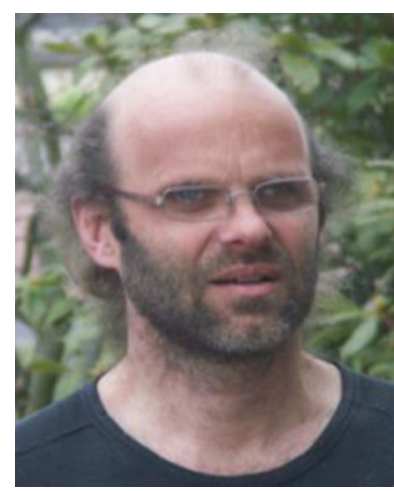

Christian Mougin was the leader of the Ecotoxicology Group (25 permanent staff) at the Joint Research Unit ECOSYS. His main interests combine the fate of contaminants (metals, pesticides, cyanotoxins, and emerging compounds) in soils, and the responses of microorganisms and macrofauna exposed to these chemicals, both in situ and through laboratory experiments. His research currently concerns the development of innovative tools for the biochemical characterizations of soils, sediments, and living microorganisms and macroorganisms, in relation to the assessment of ecosystems functioning under anthropic constraints. Christian Mougin leads the INRA's national network of Ecotoxicologists, ECOTOX (http://www6.inra. fr/ecotox). He is also in charge of French research platforms open to research in ecotoxicology, for analytical biochemistry (https://www.biochemenv.fr/) and for biological resources storage and distribution (https://www.brc4env.fr/) in the field of environment. 